\title{
Effect of diet modification in intestinal Escherichia coli population and antimicrobial resistance
}

Running title: Escherichia coli linages under diet changes

Authors: Fernanda Loayza-Villa ${ }^{1 *}$, Daniela García ${ }^{1}$, Alejandro Torres $^{2}$, Gabriel Trueba $^{1}$

\section{Contact information}

Fernanda Loayza Villa

${ }^{1}$ Microbiology Institute, Colegio de Ciencias Biológicas y Ambientales, Universidad San

Francisco de Quito. Diego de Robles y Pampite. Cumbayá- Quito. Ecuador

E-mail: mfloayzav@usfq.edu.ec

Daniela García

${ }^{1}$ Microbiology Institute, Colegio de Ciencias Biológicas y Ambientales, Universidad San

Francisco de Quito. Diego de Robles y Pampite. Cumbayá- Quito. Ecuador

E-mail: dsgarcia@estud.usfq.edu.ec

\section{Alejandro Torres}

${ }^{2}$ Carrera de medicina Veterinaria, Colegio de Ciencias de la Salud, Universidad San

Francisco de Quito. Diego de Robles y Pampite. Cumbayá- Quito. Ecuador

E mail: atorres@pronaca.com

Gabriel Trueba

${ }^{1}$ Microbiology Institute, Colegio de Ciencias Biológicas y Ambientales, Universidad San

Francisco de Quito. Diego de Robles y Pampite. Cumbayá- Quito. Ecuador

E-mail: gtureba@usfq.edu.ec

*Correspondence: mfloayzav@usfq.edu.ec 
bioRxiv preprint doi: https://doi.org/10.1101/2021.04.12.439536; this version posted April 12, 2021. The copyright holder for this preprint (which was not certified by peer review) is the author/funder, who has granted bioRxiv a license to display the preprint in perpetuity. It is made available under aCC-BY-NC-ND 4.0 International license. 


\section{ABSTRACT}

The fluctuations in the number of some intestinal bacterial lineages may be associated with increased antimicrobial resistance and disease. Adaptation to a given environment may select bacterial mutants that have reduced ability to adapt to new environments and changes in diet have been associated with alterations in microbiome taxon composition. We wanted to see the effect of diet change in linage composition and antimicrobial resistance profiles of numerically dominant $E$. coli. We subjected 50 chickens from an industrial operation (under corn-based diet supplemented with antimicrobials) to 2 antimicrobial-free diets; one based on corn and the other based on alfalfa. Fecal samples were obtained from all animals at arrival and after five weeks under different diets. Five E. coli colonies (from each sample) were subjected to genetic typing and antimicrobial susceptibility testing. We observed high diversity and high turnover rate of numerically dominant $E$. coli strains from animals from both diet groups. We did not find differences in antimicrobial resistance profiles in isolates from different diet groups. Our results suggest that there is high diversity and high turnover rate of $E$. coli strains in the intestines regardless of the diet. Chicken intestines seemed to contain many $E$. coli lineages able to thrive in different substrates. The absence of differences in antimicrobial resistance among bacteria, from animals in different diets, may indicate that the carriage of antimicrobial resistance genes does not affect the bacterial ability to adapt to different substrates. 


\section{Introduction}

A warm-blooded animal may harbor more than 10 different commensal $E$. coli lineages in the intestine, some of these lineages are numerically dominant (Lautenbach et al., 2008). The relative abundance of some $E$. coli lineages is critical because many strains carry virulence genes (Madigan, et al. 2015) and/or troublesome antimicrobial resistance (Wang, et al., 2020). The relative lineage abundance in the intestine, at any given time, may depend on their aptitude to use intestinal substrates, differential to bacteriophages, protozoan predation, and host immunity (Brito, et al., 2016; Sutton \& Hill, 2019; Tenaillon et al., 2010; Wildschutte et al., 2004)

It has been shown that different diets have a profound impact on the relative abundance of intestinal bacterial species (Chung et al., 2016; Frese et al, 2015; Gagnon et al., 2007; Niu et al., 2015; Rowland et al., 2018), however, little is known about the impact of diet change in bacterial lineages belonging to the same bacterial species. Different diets may affect the growth of different bacterial strains in the intestine; different members of the same bacterial species may have lost or acquired different metabolic properties through mutation or horizontal gene transfer (Hehemann, et al., 2010; Brito et al., 2016; Leiby and Marx, 2014). These acquired properties may facilitate or hamper the ability of some strains to use some substrates present in the diet. Genome analysis and culture experiments show that different $E$. coli strains have a variable aptitude to use different substrates (Baumler et al., 2011; Monk et al., 2013; Bouvet, et al., 2017; Barrera, et al., 2019); even a single E. coli strain passaged thousands of times in culture media produce descendants with different growth rates in different substrates (Leiby and Marx, 2014). Adaptation to 
some substrates may reduce their ability to proliferate in other substrates (Buckling, et al. 2003; Leiby and Marx, 2014).

Similarly, antimicrobial resistance genes may affect the ability of some members of the microbiota to replicate in the intestine. Genes involved in antimicrobial resistance cause fitness costs are eventually ameliorated by compensatory mutations (Andersson and Hughes, 2010; Andersson and Hughes, 2011; MacLean, et al., 2010). However, the compensatory mutations could also have fitness costs and may limit the bacterial ability to diversify and adapt to new environments or substrates (Buckling, et al. 2003). One example of this phenomenon could be the reduction of antimicrobial-resistant bacteria in the intestine after diet changes (Wu et al., 2016; Auffret et al., 2017; Liu, et al., 2019).

In this study, we aimed to observe how the $E$. coli lineages change in chicken intestines as diet is drastically altered. We analyzed the effects of a diet change in the relative frequency of numerically dominant $E$. coli strains chickens. We also assessed the effect of diet change in the frequency of antimicrobial-resistant phenotypes in numerically dominant E. coli.

\section{Methods}

\subsection{Animals}

All protocols of experimental design were approved by the ethics and biosecurity committee of the Animals Ethics Committee of Universidad San Francisco de Quito before the study.

One hundred Cobb 1day old chickens (vaccinated against Marek Gumboro, New Castle, and Bronchitis) were donated from an industrial operation where animals are fed with a corn-based diet. All animals were kept in the same diet (without 
antimicrobials) for 2 weeks before splitting the chickens into different study groups. To rule out potential effects of the surrounding environment, identical experiments were carried out in two locations (1.- USFQ 2.- farm). A randomized design was conducted with 4 homogeneous groups with 25 chicken; groups were kept separated through the experiment. In the 2 locations, one group of animals was feed with the conventional diet (D1), and the other group was feed with an alternative formula based on dry alfalfa pellets (D2), neither group received antimicrobials (Supplementary materials Table S1). This regimen was maintained for the following 5 weeks. Water was available at libitum. Each chicken was an experimental subject which has identified with a mark painted in the plumage. Fecal samples were collected from ten chickens from each pen.

\subsection{Samples and phenotypic analysis}

Fecal samples were taken from 10 randomly selected chickens which were marked for future identification. Samples were obtained from the same chickens at week 2 and week 7. Each chicken was separated in a clean cardboard box until a fecal sample was obtained in a sterile container and maintained in ice for transportation to the lab within one hour after collection. Samples were plated on MacConkey agar and incubated at $37^{\circ} \mathrm{C}$ for 18 hours. Five lactose positive colonies were selected form the plate and $\beta$-glucuronidase activity was confirmed using Chromocult Agar (Merck).

\subsection{Antimicrobial susceptibility test}

Five confirmed Escherichia coli isolates from each plate were isolated and stored at $80^{\circ} \mathrm{C}$ in TSB with $30 \%$ glycerol (Cho et al., 2007). Antimicrobial susceptibility tests were performed using AMP ampicillin (10mg), TET tetracycline (30mg), SXT trimethoprim-sulfamethoxazole (1.25/23.75mg), GEN gentamycin (10mg), AMC 
amoxicillin-clavulanic ac. (20/10mg), CIP ciprofloxacin (5mg), CHLOR chloramphenicol (30mg), IMP Imipenem (5mg), CF cefazolin (30mg), CAZ ceftazidime (30mg), FEP cefepime (30mg), and CTX cefotaxime $(30 \mathrm{mg})$ as representatives of the most used families of antibacterial drugs in health care (Eisenberg et al., 2012). The Kirby Bauer test was carried out following CLSI (Clinical \& Laboratory Standards Institute) guidelines using clinical settings for sensible or resistant phenotype interpretation.

\subsection{Strain Genotyping}

To determine whether the alfalfa diet could change the numerically dominant $E$. coli lineages, we analyzed the nucleotide sequences of the fumC gene in all isolates as published previously (Barrera, et al., 2019), and some strains showing identical sequence were submitted to full multilocus sequence typing (MLST) analysis. Briefly, the DNA from each isolate was released by the boiling method (Dashti, et al., 2009), two colonies (from each isolate) were placed in a test tube with $1 \mathrm{~mL}$ of molecular grade water, paced in a heat block at $100^{\circ} \mathrm{C}$ for $10 \mathrm{~min}$., transferred to an ice bath for 30 seconds, and centrifuged for five minutes at $1,680 \times g$ and supernatants were stored at $-20^{\circ} \mathrm{C}$ for further analysis. The fumC gene was amplified as described previously (Wirth et al., 2006). Potential clonal isolates carrying fumC4 and fumC11 were subjected to MLST to confirm clonality (alleles adk, fumC, gyrB,icd, mdh, purA, recA) as previously reported (Wirth et al., 2006). Amplicons were purified and sequenced using commercial service based on the Illumina MiSeq platform at Macrogen Inc. (Seoul, Korea). Allele and MLST were obtained using http://mlst.warwick.ac.uk/mlst/dbs/Ecoli) to define clonal relatedness (Wirth et al., 2006). 


\subsection{Statistical analysis}

Overall antimicrobial resistance was estimated at the isolate level with $95 \%$ confidence intervals. The AMR prevalence from feed treatment subgroup-collection was also estimated at the isolate level. For multidrug-resistant (MDR) estimation, isolates with resistant phenotype for three or more antimicrobial families were assigned as MDR. Prevalence estimates were carried out using the SPSS software version 24.0. The antimicrobial resistance profiles between different time points ( 2 weeks and 7 weeks) or feed source (corn-based vs. alfalfa-based) were compared by using the $x 2$ test; $p$ values $<0.05$ were considered significant. For reduction analytic dimensions, principal components analysis for categoric variables were applied using CATPCA version 2.0 by Leiden SPSS Group (Leiden University, The Netherlands). Data were analyzed in a scatter plotter using XLSTAT 2019.4.2 (Addinsoft). Sequence types were assigned to E. coli isolates with a probable clonal relation and phylogenetic analysis was conducted on Mr.Bayes Vs 3.2 bases on MCMC algorithms. Evolutionary diversity analyses were conducted using the Maximum Composite Likelihood model in MEGA $\mathrm{X}$.

\section{Results}

We found a large diversity and high turnover rates of numerically dominant $E$. coli lineages; we found that the 243 isolates had a limited number of fumC alleles $(n=47)$; fumC11 $(\mathrm{n}=72)$ and fumC4 $(\mathrm{n}=30)$ were the most common (Figure 1). We run MLST analysis in a subset of the most common alleles: fumC11 $(n=16)$ or fumC4 $(n=8)$. Most of the isolates sharing the same fumC allele belonged to different sequence types except for 5 isolates which were ST48 (fumC4) found in 4 different animals with corn diet at week 2 and 1 isolate, from a different animal in alfalfa diet, at week 7 
(Supplementary materials Table S2). Principal components analysis did not show any association between MLST profile and diet (Figure 2). Phylogenetic analysis of concatenated MLST sequences failed to show any cluster preferentially associated with any diet (Figure S1). Shannon diversity analysis of fumC alleles in both populations showed an $\mathrm{H}$ value of 2.04 for isolates from a corn-based diet and 2.43 for isolates from an alfalfa-based diet.

We did not observe differences in antimicrobial resistance phenotype in chickens in different diets (Supplementary materials Table S3) as $11.1 \%$ of the strains from chickens with a corn-based diet and $18.7 \%$ of chickens in the alfalfa-based diet, were sensitive to all antimicrobials. After 5 weeks of intervention, $32 E$ coli isolates from chickens with a conventional diet were multi-drug resistant (MDR) compared with 30 isolates from chickens with an alternative diet. The principal component analysis of antimicrobial resistance phenotypes did not show differences between diet groups (Figure 3). We observed significant differences between 2 week-chickens and 7 weekchickens, regardless of the diet $\left(\mathrm{X}_{2} ; \mathrm{p}<0,05\right)$ (Figure 3; Supplementary materials Figures S2 and S3, Table S1). The most important resistance phenotype was tetracycline (TET; 11.25\%) followed by tetracycline and cotrimoxazole (TET SXT; $8,25 \%)$. Antimicrobial resistance profiles $E$. coli were grouped in phenotype patterns. Some patterns were present in less than $1 \%$ isolates and represented the $15,75 \%$ of total phenotype patterns described (Supplementary materials Figure S2). MDR (resistance for 3 or more antimicrobials from a different family) was detected in $52.2 \%$ of $E$. coli strains. The most frequent combination was resistance to tetracycline, cotrimoxazole, chloramphenicol, and ciprofloxacin followed by tetracycline, cotrimoxazole, and ciprofloxacin combination with a 7 and $6.3 \%$ respectively. 


\section{Discussion}

We showed high diversity and a high turnover rate of dominant $E$. coli lineages associated with chicken intestinal microbiota in the chickens in both diets (Figure 1).

This finding agrees with previous reports showing high diversity and turnover rates of E. coli in human intestines (Richter, et al., 2018; Calderon, et al., 2020). We were not able to show any contribution of diet to strain diversity or turnover. We also failed to see clonal groups with a better aptitude to grow in alfalfa or corn (Supplementary materials Figure S1) which may indicate that genetically related strains may have different aptitude to grow in either of these substrates. The reason for this rapid turnover of dominant strains seems unclear but could be due to differential destruction of some E. coli lineages by bacteriophages (Sutton and Hill C., 2019), protozoa (Wildschutte et al., 2004), or host immunity (Tenaillion, 2010). It has been shown that many $E$. coli strains show the ability to adapt to different intestinal environments (generalists) as they move between different species of warm-blooded animals (Salinas et al., 2019; Moeller AH, 2018) and use diverse substrates.

We did not observe any significant variation in antimicrobial resistance in any group (Supplementary materials Figure S3), which may indicate that strains carrying antimicrobial resistance genes may not have any adaptive disadvantage when forced to grow in different substrates. Most antimicrobial resistance observed in these isolates (cotrimoxazole, chloramphenicol, and tetracycline) is widely disseminated in E. coli due to many years of antimicrobial pressure (Tedesse, 2012). Some antimicrobial resistance in E. coli emerged shortly after the invention of antimicrobials; sulfonamides were introduced in the 1930s and resistance was observed in 1950 (Tadesse et al., 2012); tetracycline was developed in 1948, resistance was observed 
in 1953 (Roberts., 1996); chloramphenicol was developed in 1947 (Tadesse et al., 2012) and resistance was found in 1955 (Watanabe, T., 1963). Plasmids carrying ARGs and bacterial chromosomes have evolved several compensatory mutations to ameliorate the fitness costs of ARGs (Andersson and Hughes, 2010; Andersson and Hughes, 2011). Contrastingly, antimicrobial resistance recently introduced in a bacterial species such as vancomycin resistance in Enterococcus faecalis and colistin resistance (mediated by the mcr-1 gene) in $E$. coli have been easily reduced by eliminating the supplementation with these type of antimicrobials (Pantosti, et al., 1999; Wang, et al, 2020).

Finally, we observed a statistically significant reduction of isolates displaying antimicrobial resistance from 2 weeks to 7 weeks, in all experimental groups (Supplementary materials Figure S3.). We also observed differences in antimicrobial phenotype in strains collected from chickens at week 2 vs at 7 weeks(figure 3 ). Previous studies have found that the proportion of antimicrobial-resistant strains is higher in 1-day- old than in older chickens (Hedman et al., 2019; Moreno et al., 2019). It has been proposed that antimicrobial-resistant $E$. coli lineages carried by 1-day chickens may gradually decrease as chickens grow (Moreno et al. 2019). This reduction may indicate that some plasmids and ARGs do cause fitness reduction as chickens grow.

The main limitation of this study is the low number of $E$. coli isolates analyzed from each chicken. Also, studying numerically dominant E. coli (Lautenbach, 2008) is an approximation to what happened to the E. coli population when the diet is changed, however, it is far less informative than a metagenomic analysis. 
The persistence of antimicrobial resistance even in the absence of antimicrobials is a serious public health concern (MacLean, et al., 2010; Andersson and Hughes, 2011). Commensal E. coli plays an important role in the transmission of antimicrobial resistance from food-animals to humans (Berg et al., 2016; Hu et al., 2016), however very little is known about the dynamics of $E$. coli population in the intestines.

\section{Declaration of Competing Interest}

The authors declare no conflict of interest.

\section{Author contributions}

GT. Principal researcher, edition of manuscript

FL and DG. All experiment performance, feeding animals, feces collection, microbiology analysis, statistics.

AT. Veterinary support and care of animals

\section{Financial support}

This project was funded by Universidad San Francisco de Quito Grant No. 11182.

The funding institutions had no participation on the project, results analysis, or manuscript edition. 


\section{References}

Auffret, M.D., Dewhurst, R.J., Duthie, C.A., Rooke, J.A., Wallace, R.J., Freeman, T.C., Stewart, R., Watson, M., Roehe, R., 2017. The rumen microbiome as a reservoir of antimicrobial resistance and pathogenicity genes is directly affected by diet in beef cattle. Microbiome 5, 159.DOI: 10.1186/s40168-017-0378-z

Andersson, D.I., Hughes, D., 2010. Antibiotic resistance and its cost: is it possible to reverse resistance? Nature Rev. Microbiol. 8, 260-271. https://doi.org/10.1038/nrmicro2319

Andersson, D.I., Hughes, D., 2011. Persistence of antibiotic resistance in bacterial populations. FEMS Microbiol. Rev. 35, 901-911. https://doi.org/10.1111/j.15746976.2011.00289.x

Barrera, S., Cardenas, P., Graham, J.P., Trueba, G., 2019. Changes in dominant Escherichia coli and antimicrobial resistance after $24 \mathrm{hr}$ in fecal matter. MicrobiologyOpen. 8, e00643. https://doi.org/10.1002/mbo3.643

Baumler, D.J., Peplinski, R.G., Reed, J.L., Glasner, J.D., Perna, N.T., 2011. The evolution of metabolic networks of E. coli. BMC Syst. Biol. 5, 182. https://doi.org/10.1186/1752-0509-5-182

Berg, E.S., Wester, A.L., Ahrenfeldt, J., Mo, S.S., Slettemeås, J.S., Steinbakk, M., Samuelsen, Grude, N., Simonsen, G.S., Løhr, I.H., Jørgensen, S.B., Tofteland, S., Lund, O., Dahle, U.R., Sunde, M., 2016. Norwegian patients and retail chicken meat share cephalosporin-resistant Escherichia coli and IncK/blaCMY2resistance plasmids. Clin. Microbiol. Infect. 23, 407.e9-407.e15. doi: 10.1016/j.cmi.2016.12.035. 
Bouvet, O., Bourdelier, E., Glodt, J., Clermont, O., Denamur, E., 2017. Diversity of the auxotrophic requirements in natural isolates of Escherichia coli. Microbiol. 163, 1260-1261. doi:10.1099/mic.0.000482

Brito, I.L., Yilmaz, S., Huang, K., Xu, L., Jupiter, S.D., Jenkins, A.P., Naisilisili, W., Tamminen, M., Smillie, C.S., Wortman J.R., Birren, B.W., Xavier, R.J., Blainey, P.C., Singh AK, Gevers, D., Alm, E.J., 2016. Mobile genes in the human microbiome are structured from global to individual scales. Nature 535, 435-439. https://doi.org/10.1038/nature18927

Buckling, A., Wills, M,A., Colegrave, N., 2003. Adaptation limits diversification of experimental bacterial populations. Science 302, 2107-2109.

doi:10.1126/science. 1088848

Calderon D., Cardenas P., Graham J., Trueba G., 2020. A longitudinal study of E. coli lineages and antimicrobial resistance in Ecuadorian children. bioRxiv doi.org/10.1101/2020.11.09.375931;

Chung, W.S.F., Walker, A.W., Louis, P., Parkhill, J., Vermeiren, J., Bosscher, D., Duncan, S.H., Flint, H.J., 2016. Modulation of the human gut microbiota by dietary fibres occurs at the species level. BMC Biol. 14, 1-13.

https://doi.org/10.1186/s12915-015-0224-3

Dashti, A.A., Jadaon, M.M., Abdulsamad, A.M., Dashti, H.M., 2009. Heat treatment of bacteria: A simple method of DNA extraction for molecular techniques. Kuwait Med. J. 41, 117-122.

https://www.researchgate.net/publication/266888615_Heat_Treatment_of_Bacteri a_A_Simple_Method_of_DNA_Extraction_for_Molecular_Techniques/stats 
Eisenberg, J.N.S., Goldstick, J., Cevallos, W., Trueba, G., Levy, K., Scott, J., Percha, B., Segovia, R., Ponce, K., Hubbard, A., Marrs, C., Foxman, B., Smith, D.L., Trostle, J., (2012). In-roads to the spread of antibiotic resistance: regional patterns of microbial transmission in northern coastal Ecuador. J R Soc Interface 9, 1029-1039. https://doi.org/10.1098/rsif.2011.0499

Frese, S.A., Parker, K., Calvert, C.C., Mills, D.A., 2015. Diet shapes the gut microbiome of pigs during nursing and weaning. Microbiome 3, 1-10. https://doi.org/10.1186/s40168-015-0091-8

Hedman, H.D., Eisenberg, J.N.S., Vasco, K.A., Blair, C.N., Trueba, G., Berrocal, V.J., and Zhang, L., 2019. High prevalence of extended-spectrum beta-lactamase ctxm-producing Escherichia coli in small-scale poultry farming in rural Ecuador. Am. J. Trop. Med. Hyg. 100, 374-376. https://doi.org/10.4269/ajtmh.18-0173

Hehemann, J.H., Correc, G., Barbeyron, T., Helbert, W., Czjzek, M., Michel, G., 2010. Transfer of carbohydrate-active enzymes from marine bacteria to Japanese gut microbiota. Nature 464, 908-912. https:// doi:10.1038/nature08937

Hu, Y., Yang, X., Li, J., Lv, N., Liu, F., Wu, J., Lin, I.Y., Wu, N., Weimer, B.C., Gao, G.F., Liu Y., Zhu, B. (2016). The bacterial mobile resistome transfer network connecting the animal and human microbiomes. Appl. Environ. Microbiol. 82, 6672-6681. https://doi.org/10.1128/AEM.01802-16

Lautenbach, E., Bilker, W.B., Tolomeo, P., \& Maslow, J.N., 2008. Impact of diversity of colonizing strains on strategies for sampling Escherichia coli from fecal specimens. J. Clin. Microbiol. 46, 3094-3096. https://doi.org/10.1128/JCM.00945-08 
Leiby, N., Marx, C.J., 2014. Metabolic erosion primarily through mutation accumulation, and not tradeoffs, drives limited evolution of substrate specificity in Escherichia coli. PLoS Biol. 12, e1001789.

https://doi.org/10.1371/journal.pbio.1001789

Liu, J., Taft, D.H., Maldonado-Gomez, M.X., Johnson, D., Treiber, M.L., Lemay, D.G., DePeters, E.J., Mills, D.A., 2019. The fecal resistome of dairy cattle is associated with diet during nursing. Nat. Commun. 10, 4406. https://doi.org/10.1038/s41467019-12111-x

MacLean, R.C., Hall, A.R., Perron, G.G., Buckling, A., 2010. The population genetics of antibiotic resistance: integrating molecular mechanisms and treatment contexts. Nat Rev Genet.11, 405-414. doi:10.1038/nrg2778

Madigan, T., Johnson, J.R., Clabots, C., Johnston, B.D., Porter, S.B., Slater, B.S., Banerjee, R., 2015. Extensive household outbreak of urinary tract infection and intestinal colonization due to extended-spectrum $\beta$-Lactamase-producing Escherichia coli sequence type 131. Clin. Infect. Dis. 61, e5-e12. doi:10.1093/cid/civ273

Moeller A.H., Suzuki, T.A., Phifer-Rixey, M., Nachman, M.W., 2018.Transmission modes of the mammalian gut microbiota. Science. 362, 453-457. https//doi:10.1126/science.aat7164

Monk, J.M., Charusanti, P., Aziz, R.K., Lerman, J.A., Premyodhin, N., Orth, J.D., Feist, A.M., Palsson, B., 2013. Genome-scale metabolic reconstructions of multiple Escherichia coli strains highlight strain-specific adaptations to nutritional environments. PNAS USA, 110, 20338-20343. 
https://doi.org/10.1073/pnas. 1307797110

Moreno, M.A., García-Soto, S., Hernández, M., Bárcena, C., Rodríguez-Lázaro, D., Ugarte-Ruíz, M., Domínguez, L., 2019. Day-old chicks are a source of antimicrobial resistant bacteria for laying hen farms. Vet. Microbiol. 230, 221-227. https://doi.org/10.1016/j.vetmic.2019.02.007

Niu, Q., Li, P., Hao, S., Zhang, Y., Kim, S. W., Li, H., Ma, X., Gao, S., He, L., Wu, W., Huang, X., Hua, J., Zhou, B., Huang, R., 2015. Dynamic distribution of the gut microbiota and the relationship with apparent crude fiber digestibility and growth stages in pigs. Sci. Rep. 5, 9938. https://doi.org/10.1038/srep09938

Pantosti, A., Grosso, M. Del, Tagliabue, S., Macrì, A., Caprioli, A., 1999. Decrease of vancomycin-resistant enterococci in poultry meat after avoparcin ban. Lancet 354, 741-742. https://doi.org/10.1016/S0140-6736(99)02395-8

Richter, T.K.S., Hazen, T.H., Lam, D., Coles, C., Seidman, J., You, Y., Silergeld, E., Fraser, C., Rasko, D., 2018. Temporal variability of Escherichia coli diversity in the gastrointestinal tracts of tanzanian children with and without exposure to antibiotics. MSphere 3, e00558-18. doi:10.1128/mSphere.00558-18

Roberts, M.C., 1996. Tetracycline resistance determinants: mechanisms of action, regulation of expression, genetic mobility, and distribution. FEMS Microbiol. Rev. 19, 1-24. 10.1111/j.1574-6976.1996.tb00251.x

Rowland, I., Gibson, G., Heinken, A., Scott, K., Swann, J., Thiele, I., Tuohy, K., 2018. Gut microbiota functions: metabolism of nutrients and other food components. Eur. J. Nutr. 57, 1-24. https://doi.org/10.1007/s00394-017-1445-8 
Salinas, L., Cárdenas, P., Johnson, T.J., Vasco, K., Graham, J., Trueba, G., 2019. Diverse commensal Escherichia coli clones and plasmids disseminate antimicrobial resistance genes in domestic animals and children in a semirural community in Ecuador. mSphere 4, e00316-19.

https://doi.org/10.1128/mSphere.00316-19

Sutton, T.D.S., Hill, C., 2019. Gut bacteriophage: current understanding and challenges. Front Endocrinol (Lausanne). 10, 784. https://doi:10.3389/fendo.2019.00784

Tadesse, D.A., Zhao, S., Tong, E., Ayers, S., Singh, A., Bartholomew, M.J., McDermott, P.F., 2012. Antimicrobial drug resistance in Escherichia coli from humans and food animals, United States, 1950-2002. Emerg. Infect. Dis. 18, 741749. https://doi.org/10.3201/eid1805.111153.

Tenaillon, O., Skurnik, D., Picard, B., Denamur, E., 2010.The population genetics of commensal Escherichia coli. Nat Rev Microbiol. 8, 207-217. https://www.nature.com/articles/nrmicro2298

Wang, Y., Xu, C., Zhang, R., Chen, Y., Shen, Y., Hu, F., Liu, D., Lu, J., Guo, Y., Xia, X., Jiang, J., Wang, X., Fu, Y., Yang, L., Wang, J., Li, J., Cai, C., Yin, D., Che, J., Fan, R., Wang, Y., Qing, Y., Li, Y., Liao, K., Chen, H., Zou, M., Liang, L., Tang, J., Shen, Z., Wang, S., Yang, X., Wu, C., Xu, S., Walsh, T.R., Shen, J., 2020. Changes in colistin resistance and mcr-1 abundance in Escherichia coli of animal and human origins following the ban of colistin-positive additives in China: an epidemiological comparative study. Lancet Infect. S1473-3099(20)30149-3. https://doi.org/10.1016/S14733099(20)30149-3

Watanabe, T., 1963. Infective heredity of multiple drug resistance in bacteria. 
Bacteriol. Rev. 27, 87-115. https://mmbr.asm.org/content/27/1/87

Wildschutte, H., Wolfe, D.M., Tamewitz, A., Lawrence, J.G., 2004. Protozoan

predation, diversifying selection, and the evolution of antigenic diversity in

Salmonella. PNAS USA 101, 10644-10649. doi:10.1073/pnas.0404028101

Wirth, T., Falush, D., Lan, R., Colles, F., Mensa, P., Wieler, L H., Karch, H., Reeves, P.R., Maiden, M.C., Ochman, H., Achtman, M., 2006. Sex and virulence in Escherichia coli: An evolutionary perspective. Mol. Microbiol. 60, 1136-1151. https://doi.org/10.1111/j.1365-2958.2006.05172.x

Wu, G., Zhang, C., Wang, J., Zhang, F., Wang, R., Shen, J., Wang, L., Pang, X., Zhang, X., Zhao, L., Zhang, M., 2016. Diminution of the gut resistome after a gut microbiota-targeted dietary intervention in obese children. Sci. Rep. 6, 24030. doi: 10.1038/srep24030. PMID: 27044409; PMCID: PMC4820771. 


\section{Figures and figure legends}

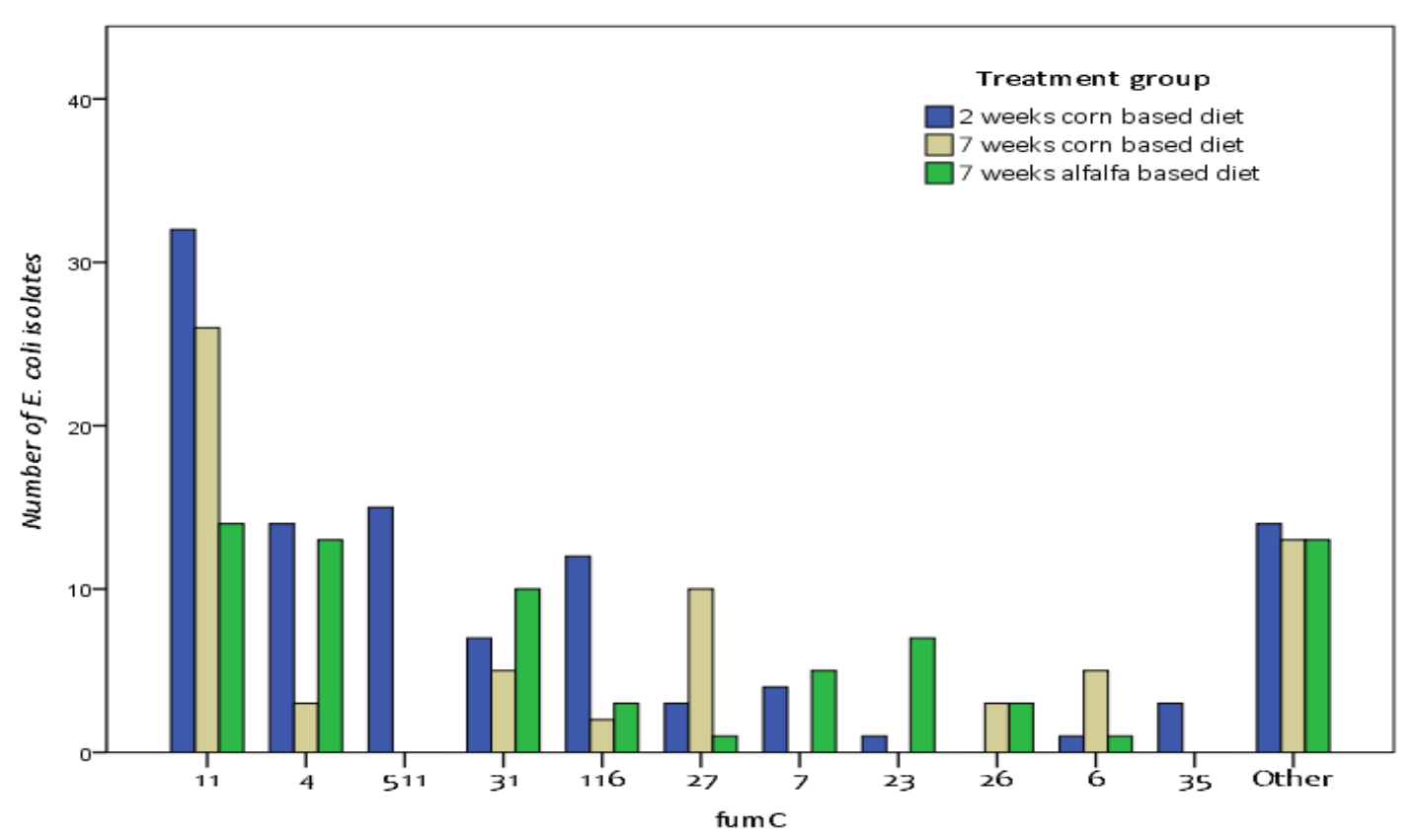

Figure 1. The number of Escherichia coli isolates carrying different fumC alleles from study groups. $E$. coli isolates $(\mathrm{n}=243)$ that were isolated from chicken feces; 106 from 2 weeks old chickens (blue bars); from 67 with corn-based feed (yellow bars) and 70 from chickens with alfalfa-based feed (green bars). Alleles with less than $1 \%$ of frequency where merge in "Other" category. 


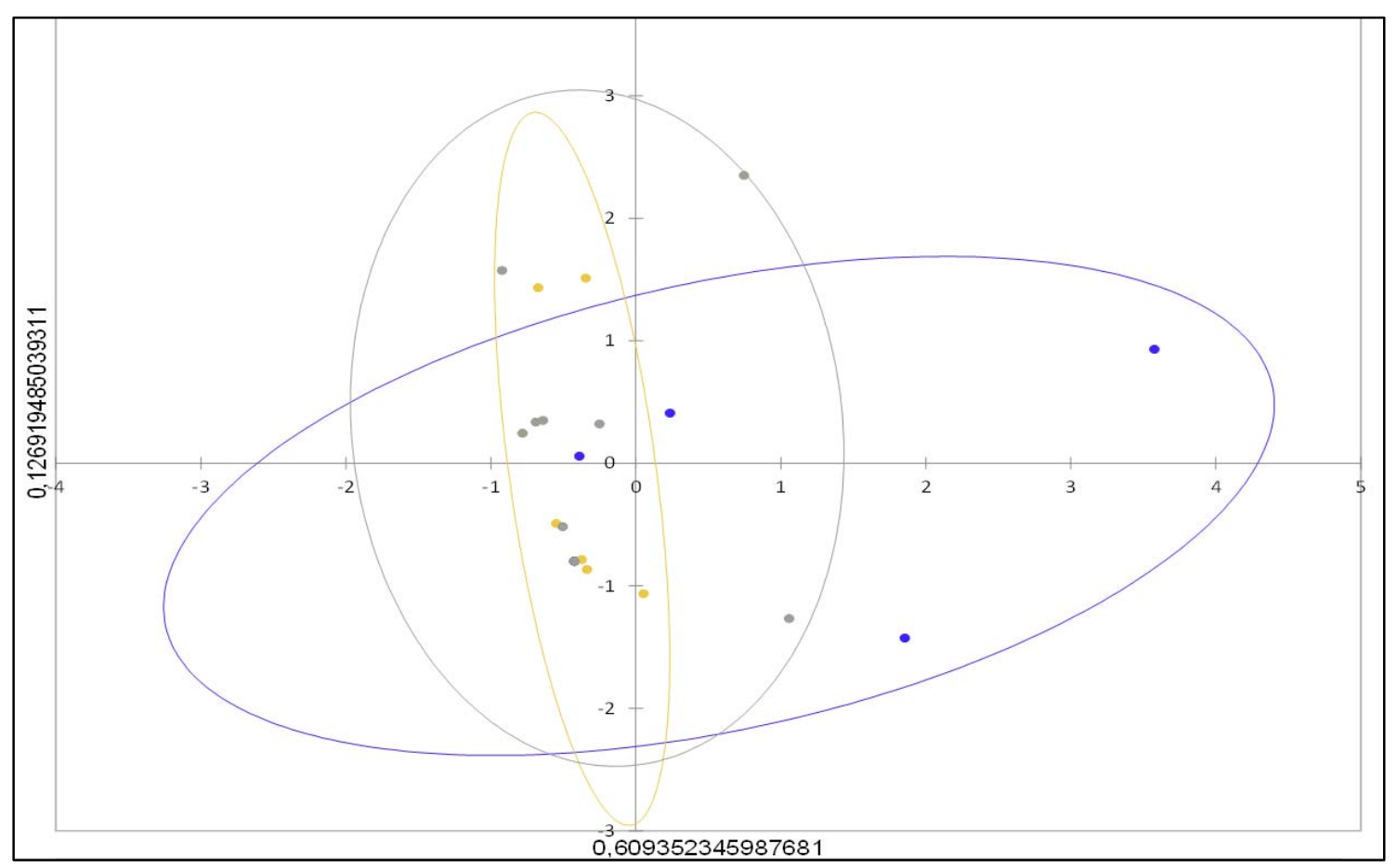

Figure 2: Principal components of 7 housekeeping genes profile of selected Escherichia coli isolates. 1. Blue dots represent isolates from 2 weeks chicken (basal), 2. Yellow dots represent isolated from 7-week chickens feed with a cornbased diet. 3. Grey dot represents isolates from 7-week chickens feed with an alfalfabased diet. Confidence intervals $95 \%$ based on chi-square are graph in colored ellipses according to the origin of isolates. 


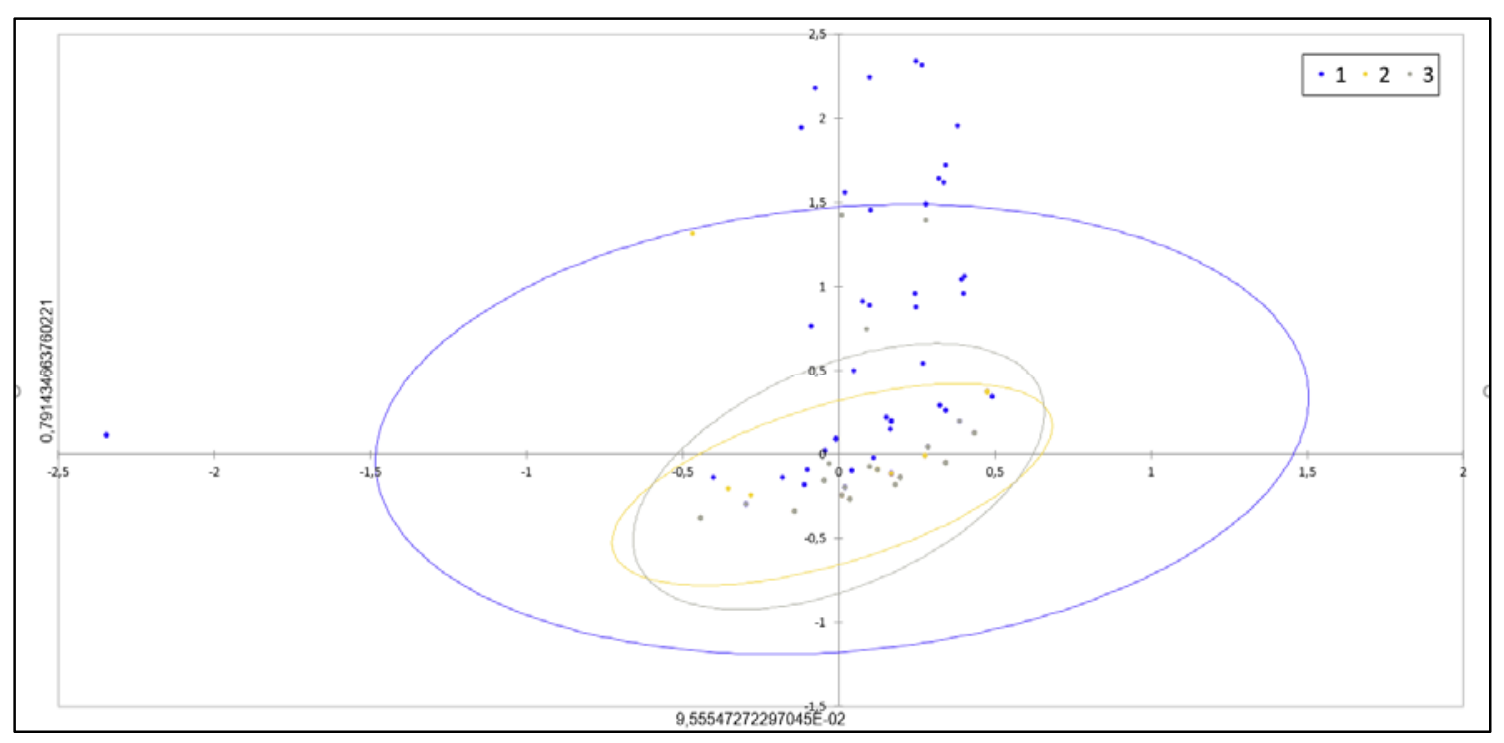

Figure 3. Principal components of phenotypic antimicrobial resistance profiles of 389 Escherichia coli isolates. 1. Blue dots represent isolates from 2 weeks chicken (basal), 2. Yellow dots represent isolated from 7-week chickens feed with a corn-based diet. 3. Grey dot represents isolates from 7-week chickens feed with an alfalfa-based diet. Confidence intervals $95 \%$ based on chi-square are graph in colored ellipses according to the origin of isolates. 
bioRxiv preprint doi: https://doi.org/10.1101/2021.04.12.439536; this version posted April 12, 2021. The copyright holder for this preprint (which

was not certified by peer review) is the author/funder, who has granted bioRxiv a license to display the preprint in perpetuity. It is made available under aCC-BY-NC-ND 4.0 International license.

\section{Supplementary Materials}

\section{Tables}

Table S1. Detailed analysis of diet formulation administered in each treatment groups

\begin{tabular}{|l|l|l|}
\hline & Corn-based Diet & Alfalfa-based Diet \\
\hline Alfalfa pellets & $0 \%$ & $100 \%$ \\
\hline Corn & $40 \%$ & $0 \%$ \\
\hline Sorgo & $5 \%$ & $0 \%$ \\
\hline Wheat & $12 \%$ & $0 \%$ \\
\hline Rice & $3 \%$ & $0 \%$ \\
\hline Soy & $5 \%$ & $0 \%$ \\
\hline Soy paste & $24 \%$ & $0 \%$ \\
\hline Fish floor & $3 \%$ & $0 \%$ \\
\hline Bird floor & $3 \%$ & $0 \%$ \\
\hline Salt & $0.376 \%$ & $0 \%$ \\
\hline
\end{tabular}


Table S2.- Sequence type from Escherichia coli strains. Alleles are described from each isolate. Treatment group: 1) 2-weeks-old chickens with a corn-based diet. 2) 7weeks-old chicken with a corn-based diet and 3) 7-weeks-old chicken with an alfalfabased diet. Also, phenotypic patterns of antimicrobial susceptibility from each isolate are described according to the antimicrobial discs used in Kirby Bauer test: AMP ampicillin $(10 \mathrm{mg})$, TET tetracycline $(30 \mathrm{mg})$, SXT trimethoprim-sulfamethoxazole (1.25/23.75mg), GEN gentamycin (10mg), AMC amoxicillin-clavulanic ac. (20/10mg), CIP ciprofloxacin (5mg), CHLOR chloramphenicol (30mg), IMP Imipenem $(5 \mathrm{mg})$, CF cefazolin (30mg), CAZ ceftazidime (30mg), FEP cefepime $(30 \mathrm{mg})$, and CTX cefotaxime $(30 \mathrm{mg})$.

\begin{tabular}{|c|c|c|c|c|c|c|c|c|c|c|}
\hline & & & \multicolumn{6}{|c|}{ Alleles } & \multicolumn{2}{|r|}{ MLST } \\
\hline $\begin{array}{l}\text { Treat } \\
\text { ment } \\
\text { group }\end{array}$ & sample ID & Antimicrobial phenotype & adk & fumC & gyrB & icd & $\mathrm{mdh}$ & purA & recA & ST \\
\hline 2 & 3.2 & $\begin{array}{l}\text { AMC TET SXT CHLOR } \\
\text { AMP CIP }\end{array}$ & -196 & 11 & 55 & 101 & 113 & 40 & 38 & ni \\
\hline 1 & 3.3 & $\begin{array}{l}\text { TET SXT CHLOR AMP } \\
\text { CIP }\end{array}$ & 6 & 11 & 3 & 18 & 70 & 8 & 6 & ni \\
\hline 1 & 8.2 & TET SXT CIP CHLOR & 6 & 11 & 4 & 8 & 8 & 8 & 2 & 48 \\
\hline 2 & 8.3 & TET SXT CHLOR CIP & -199 & 11 & 4 & 8 & 8 & 1 & 4 & ni \\
\hline 3 & 13.3 & TET CHLOR AMP & 6 & 4 & 4 & 10 & 7 & 8 & 14 & ni \\
\hline 3 & 13.1 & $\begin{array}{l}\text { AMC TET CTX FEP } \\
\text { SXT AMP }\end{array}$ & 6 & 11 & 14 & 16 & 24 & 8 & 6 & 2497 \\
\hline 3 & 15.4 & TET IMP AMP & 6 & 11 & 4 & 8 & 8 & 8 & 2 & 48 \\
\hline 1 & 14.1 & TET & 6 & 4 & 14 & 16 & 24 & 8 & 14 & 155 \\
\hline 1 & 16.3 & $\begin{array}{l}\text { AMC TET SXT AMP } \\
\text { CIP CF }\end{array}$ & 6 & 4 & 4 & 16 & 24 & 8 & 14 & 58 \\
\hline 3 & 16.2 & TET SXT & 10 & 4 & 14 & 16 & 24 & 62 & 2 & ni \\
\hline 1 & 18.3 & TET SXT CIP AMP GN & 6 & 11 & 4 & 8 & 8 & 8 & 2 & 48 \\
\hline 3 & 18.2 & TET CIP & 10 & 4 & 4 & 8 & 8 & 8 & 4 & ni \\
\hline 3 & 20.1 & TET AMP & 10 & 11 & 4 & 10 & 7 & 8 & 2 & 2705 \\
\hline 1 & 20.3 & TET SXT AMP CIP & 6 & 4 & 14 & 1 & 20 & 62 & 7 & 345 \\
\hline 2 & 21.4 & TET AMP & 6 & 11 & 5 & 8 & 8 & 8 & 2 & 6396 \\
\hline 2 & 24.2 & TET SXT CIP & 10 & 4 & 4 & 10 & 24 & 8 & 14 & 5519 \\
\hline 2 & 25.5 & TET CHLOR CIP & 24 & 11 & 4 & 8 & 8 & 8 & 73 & 73 \\
\hline 2 & 26.4 & TET SXT CIP & 10 & 4 & 4 & 8 & 8 & 8 & 14 & 2883 \\
\hline 2 & 26.5 & TET SXT CIP & 6 & 11 & 4 & 8 & 7 & 8 & 2 & 5224 \\
\hline 1 & 27.4 & $\begin{array}{l}\text { TET SXT CIP AMP GN } \\
\text { IMP CHLOR }\end{array}$ & 6 & 11 & 4 & 8 & 8 & 8 & 2 & 48 \\
\hline 1 & 28.5 & $\begin{array}{l}\text { AMC TET CTX CAZ } \\
\text { SXT AMP CIP CF }\end{array}$ & 10 & 11 & 14 & 8 & 8 & 8 & 313 & 4536 \\
\hline 1 & 29.1 & TET SXT CIP & 6 & 11 & 4 & 8 & 8 & 8 & 2 & 48 \\
\hline 2 & 30.3 & TET SXT CHLOR AMP & 6 & 11 & 14 & 10 & 7 & 8 & 2 & ni \\
\hline 3 & 35.1 & TET SXT CIP & 24 & 11 & 4 & 8 & 8 & 8 & 2 & 43 \\
\hline 3 & 39.1 & TET SXT CIP & 10 & 11 & 4 & 10 & 8 & 8 & 2 & 4704 \\
\hline 3 & 40.4 & TET SXT & 10 & 11 & 4 & 8 & 8 & 1 & 2 & 1585 \\
\hline
\end{tabular}

ni.- no identified 
Table S3. Frequency of resistance phenotype analyzed by the antimicrobial disc in Kirby Bauer test. In first column antimicrobial used are listed: AMP ampicillin (10mg), TET tetracycline (30mg), SXT trimethoprim-sulfamethoxazole (1.25/23.75mg), GEN gentamycin (10mg), AMC amoxicillin-clavulanic ac. (20/10mg), CIP ciprofloxacin (5mg), CHLOR chloramphenicol (30mg), IMP Imipenem (5mg), CF cefazolin (30mg), CAZ ceftazidime (30mg), FEP cefepime $(30 \mathrm{mg})$, and CTX cefotaxime $(30 \mathrm{mg})$. The letter after the percentage of resistant isolates in each group means the category according to X2 test. The same letter in three columns means that the proportions are not significantly different under the 0.05 level.

\section{Treatment group}

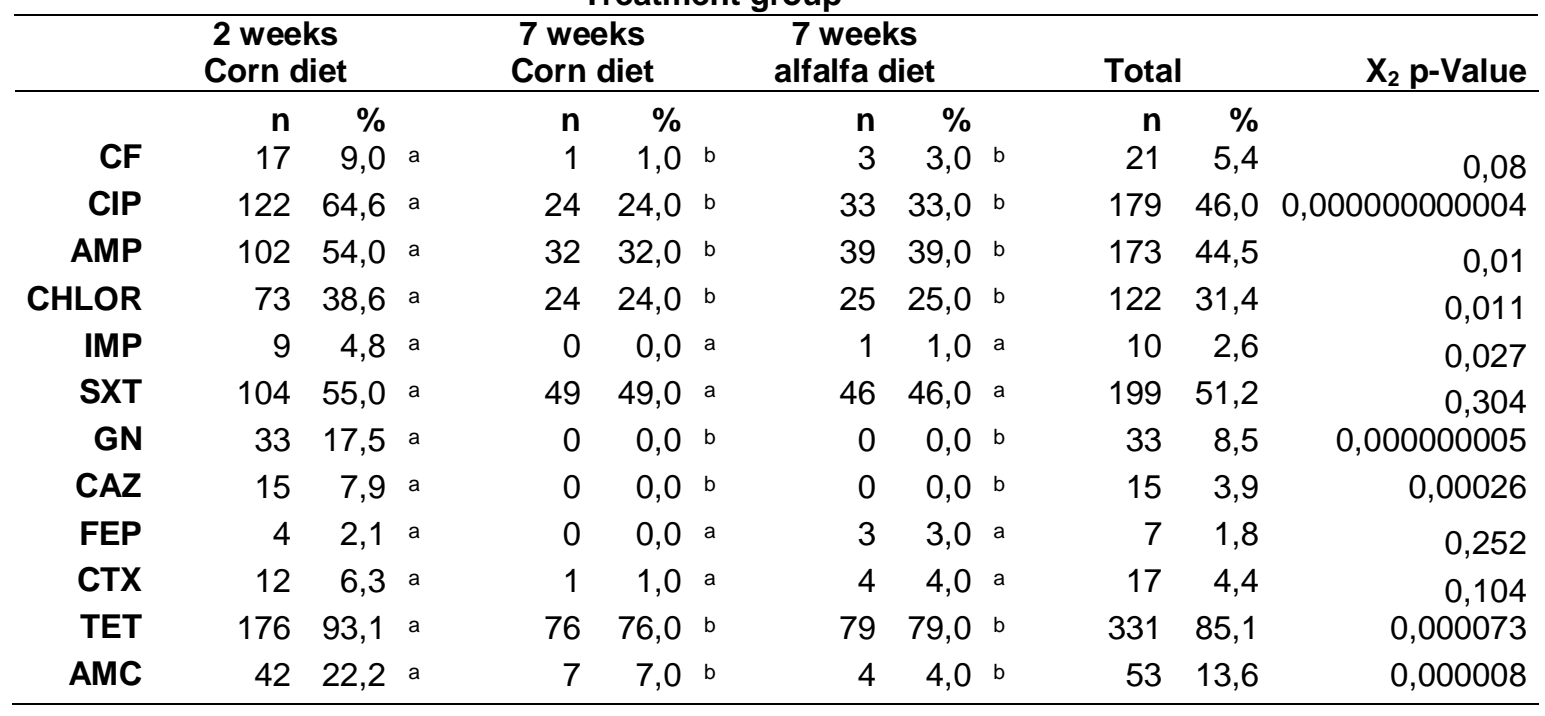




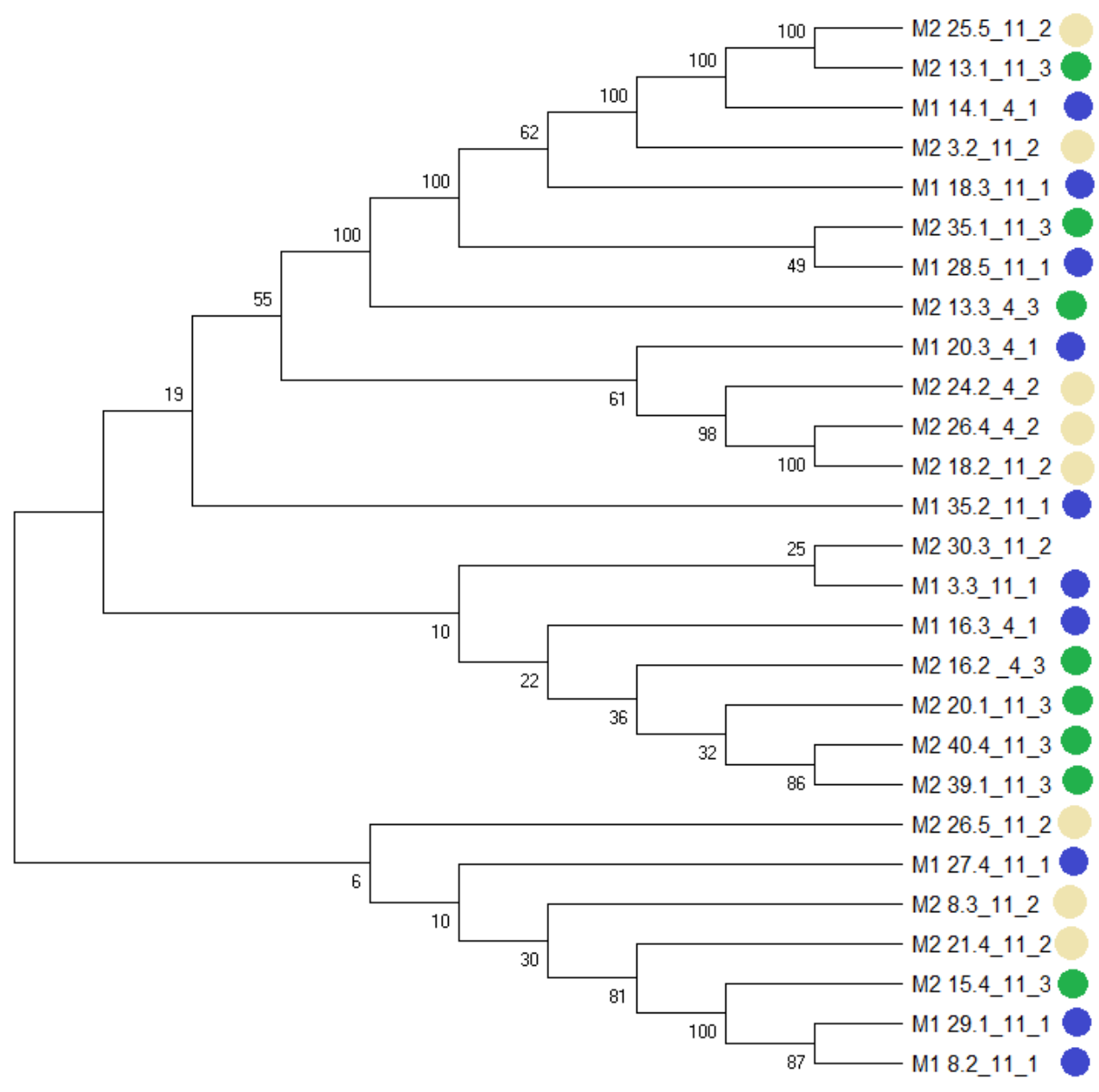

Figure S1. Bayesian phylogenetic tree was constructed from seven concatenated housekeeping genes used for MLST E. coli analysis. 1. Isolates from 2-week-old chicken (blue dots), 2. Isolates from 7-years-old chickens feed with a conventional corn-based formula (yellow dots) and 3. Isolates from 7-years-old chickens feed with an alfalfa-based formula (green dots). The identification label describes M1.-2 weeks old chicken, M2.- 7 weeks old chicken, followed by the ID number of each animal. After the underscore, fumC allele 4 or 11 are described. The last number after the second underscore confirms the treatment group. 


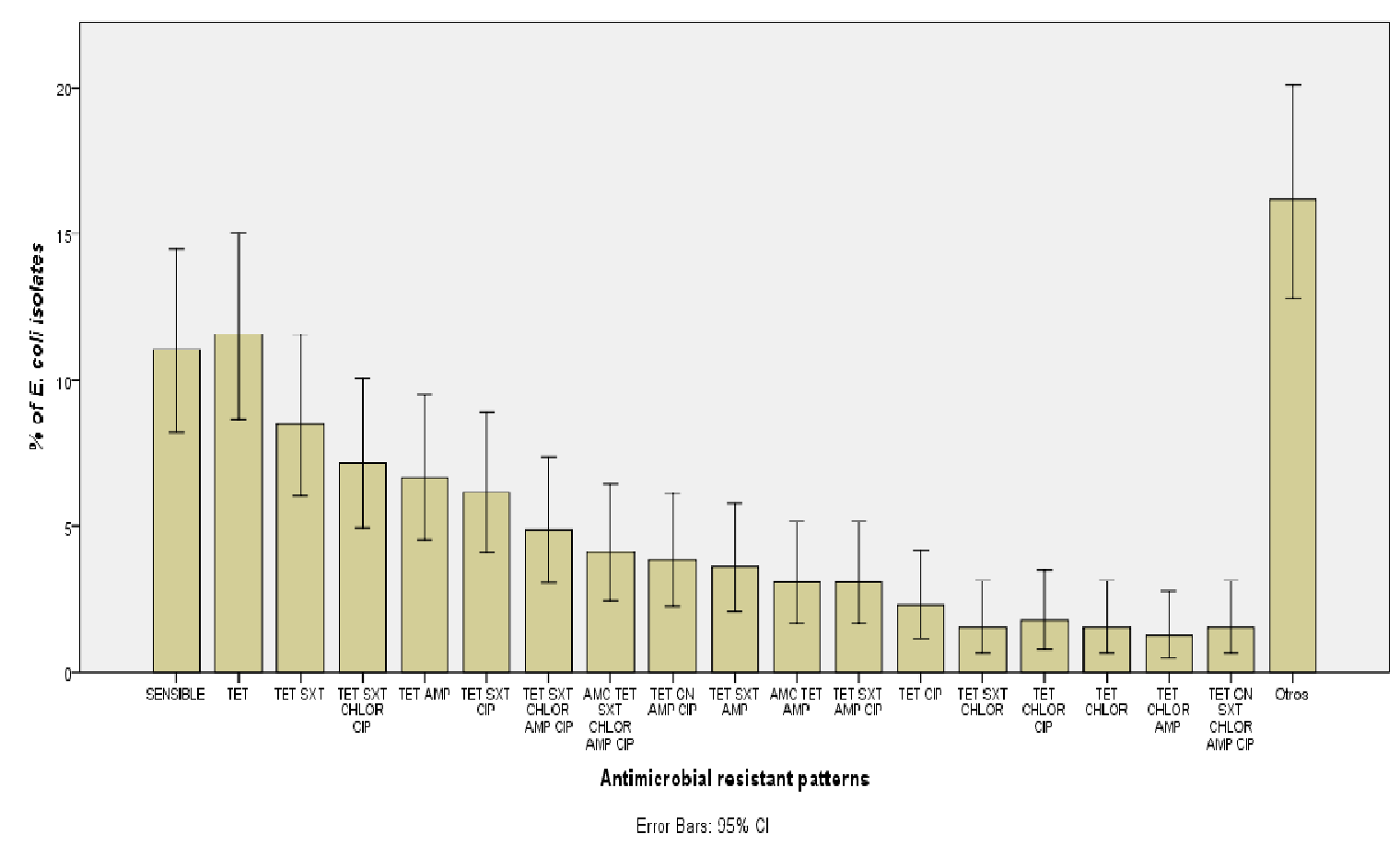

Figure S2. Overall frequency distribution of antimicrobial susceptibility patterns from 389 commensal Escherichia coli isolated from chicken feces in Mac Conkey Lactosa plates; 189 from 2 weeks old chickens, 100 from 7 weeks old chicken feed with a cornbased diet, and 1007 week old chickens that were feed with alfalfa-based formula. Any diet has antimicrobial supplements. Kirby Bauer technique was performed for antimicrobial susceptibility testing (AST). AMP ampicillin (10mg), TET tetracycline (30mg), SXT trimethoprim-sulfamethoxazole (1.25/23.75mg), GEN gentamycin (10mg), AMC amoxicillin-clavulanic ac. (20/10mg), CIP ciprofloxacin (5mg), CHLOR chloramphenicol (30mg), IMP Imipenem (5mg), CF cefazolin (30mg), CAZ ceftazidime (30mg), FEP cefepime (30mg), and CTX cefotaxime (30mg). 


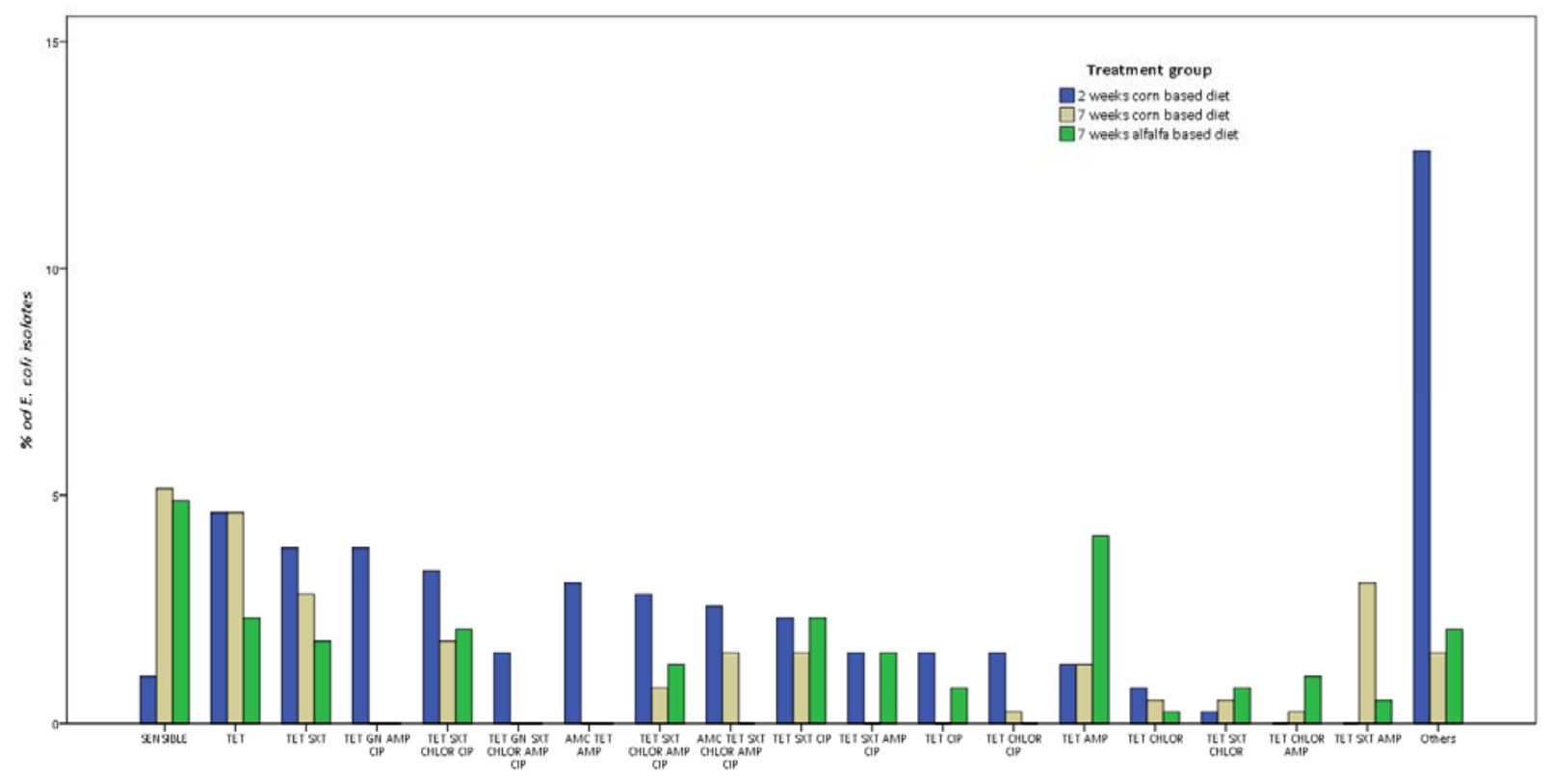

Figure S3. The number of $E$. coli isolates showing a specific phenotypic pattern of antimicrobial resistance. E. coli isolates $(n=389)$ were isolated from chicken feces in Mac Conkey Lactosa plates; 189 from 2 weeks old chickens (blue bars) and 200 from 7 weeks old chickens, separated by feed administration 100 with corn-based feed (green bars), and 100 from chickens with alfalfa-based feed (yellow bars). Kirby Bauer technique was performed for antimicrobial susceptibility testing (AST) and antimicrobial-resistant phenotype patterns are shown. Patterns with less than $1 \%$ where merge in "Other" category. AMP ampicillin (10mg), TET tetracycline $(30 \mathrm{mg})$, SXT trimethoprim-sulfamethoxazole (1.25/23.75mg), GEN gentamycin (10mg), AMC amoxicillin-clavulanic ac. (20/10mg), CIP ciprofloxacin (5mg), CHLOR chloramphenicol (30mg), IMP Imipenem (5mg), CF cefazolin (30mg), CAZ ceftazidime (30mg), FEP cefepime (30mg), and CTX cefotaxime (30mg). 